\title{
Using Barbour's Ephemeris Time, and Padmanabhan's Inflaton Value, plus Will's Massive Graviton Velocity to Isolate Rest Energy of Massive Graviton as Compared to Racetrack Inflation Results of Graviton Physics and Modified Wheeler de Witt Results of Wormhole Physics
}

\author{
Andrew Walcott Beckwith \\ College of Physics, Chongqing University Huxi Campus, Chongqing, China \\ Email: Rwill9955b@gmail.com
}

How to cite this paper: Beckwith, A.W. (2017) Using Barbour's Ephemeris Time, and Padmanabhan's Inflaton Value, plus Will's Massive Graviton Velocity to Isolate Rest Energy of Massive Graviton as Compared to Racetrack Inflation Results of Graviton Physics and Modified Wheeler de Witt Results of Wormhole Physics. Journal of High Energy Physics, Gravitation and Cosmology, 3, 754-775. https://doi.org/10.4236/jhepgc.2017.34056

Received: September 11, 2017

Accepted: October 27, 2017

Published: October 30, 2017

Copyright $\odot 2017$ by author and Scientific Research Publishing Inc. This work is licensed under the Creative Commons Attribution International License (CC BY 4.0).

http://creativecommons.org/licenses/by/4.0/

\section{(c) (i) Open Access}

\begin{abstract}
The idea is to identify via ephemeris time as given by Barbour and an inflaton field as given by Padmanabhan, for scale factor proportional to time to the alpha power and a velocity given by Will for massive gravitons, an initial energy for a massive graviton in space-time. The spatial values for the graviton production could be from the Planckian to Electro weak regime, with a nod to using a worm hole from a prior to a present universe as a delivery font for gravitational energy, as an information carrying bridge from prior universe "information settings" to the present space-time. The number of Gravitons will be set as $\mathrm{N}$, and the initial time, as a tie in with Barbour's ephemeris time, a constant times Planck time. In setting up the positions, as input into the positions and distributions of gravitons in our model, we will compare results as could be generated by Racetrack inflation, for presumed position of relic gravitons when just produced in the universe, as compared with results given by an adaptation of an argument presented by Crowell, in a modification of the Wheeler de Witt equation he gave germane to worm hole physics. In addition, with this presentation we will discuss entropy generation via graviton production. And compare that with semi classical arguments, as well as Brane-anti brane combinations. The idea will be to in all of this to re set the particulars of massive gravity in such a way as to revisit the outstanding problem of massive gravity: Its predictions do not match those of general relativity
\end{abstract}


in the limit when a massive graviton mass approaches zero\{ \displaystyle $\mathrm{m} \backslash$ to $0\}$. In particular, while at small scales, Newton's gravitational law is recovered, the bending of light is only three quarters of the result Albert Einstein obtained in general relativity

\section{Keywords}

Ephemeris Time, Inflaton, Massive Graviton

\section{Introduction}

One of the inquiries as to graviton physics, is to ascertain how to gauge the real actual energy of a "massive" graviton. The reason for doing this, is due to the well known physics problem of how the bending of light by massive gravitons via the Planet Mercury is 3/4th that of the actual results seen in GR i.e. In the 1970s, van Dam and Veltman [1] and Zakharov [2] discovered a property of Fierz-Pauli massive gravity. Its predictions do not match those of general relativity in the limit when a massive graviton mass approaches zero\{ $\{$ displaystyle $\mathrm{m} \backslash$ to 0\}. In particular, while at small scales, Newton's gravitational law is recovered, the bending of light is only three quarters of the result Albert Einstein obtained in general relativity. This is known as the $v D V Z$ discontinuity. [3] [4] [5] gives a summary on page 94 as to the details of the Vainstein solution which in the limit of non-linearized gravity, in its Equation (2.184) give a partial solution via a solution with a screening Yukawa type of potential as to what happens, when the mass of a graviton, approaches zero.

We will try to avoid using Yukawa style screening, and our start will be to ascertain an actual "rest energy" of a "massive" graviton, where we may be able to recover the limit behavior we want as $m_{g} \rightarrow 0$. To do this, we will be using [6] by Barbour, but not in the sense of [7] [8]. In addition, [9] will be employed to obtain a velocity for a massive graviton, which has the energy $\mathrm{E}$ term we will attempt to isolate. [10] has the inflaton, we will be using which we will utilize for early universe kinetic energy contributions.

Afterwards, in $2^{\text {nd }}$ part of the manuscript we will briefly state some phenomenological consequences of what we have derived, and then detail those findings with possible consequences to the problem of early universe graviton generation and of an average energy, for a graviton, resulting from early universe production of gravitons.

The $3^{\text {rd }}$ part of the manuscript introduces in a general sense the problem of the position of gravitons, as assumed to be evaluated.

In the $4^{\text {th }}$ part of the manuscript, we will allude to racetrack inflation [11] as far as its connections to graviton physics, as well as non standard treatments of the WdW equation which were written up by Crowell, in 2005 [12]. 
The $5^{\text {th }}$ part of this manuscript will be a discussion between different choices of entropy.

The $6^{\text {th }}$ part of this manuscript is a review of applications of non-standard treatments of the WdW equation which were written up by Crowell, in 2005 [12].

Our conclusion will be a wrap up of our findings plus a prospectus as we see it as to what to possibly expect next, and to ascertain what may be fruitful lines of inquiry. As to the originally stated problem of fixing massive gravitons, only $3 / 4$ of the angular deviation of light about the planet mercury is given.

\section{Barbour's Ephemeris Time, and Padmanabhan's Inflaton Value, Plus Will's Massive Graviton Velocity to Isolate Rest Energy of Massive Graviton}

From the use of [6], we have a statement of Ephemeris Time which is

$$
\delta t=\sqrt{\frac{\sum_{i=1}^{N} m_{i} \cdot(\delta d)_{i}^{2}}{2 \cdot(E-V)}}
$$

$m_{i}$ refers to the mass of an $i$ th body, $(\delta d)_{i}$ is position of $i$ th body, and $(E-V)$ is kinetic energy of the system we are analyzing. We will use the construction given in [10] to construct the relevant kinetic energy of the system we are trying to analyze to make our point.

$$
\begin{aligned}
& a \approx a_{\min } t^{\gamma} \\
& \Leftrightarrow \phi \approx \sqrt{\frac{\gamma}{4 \pi G}} \cdot \ln \left\{\sqrt{\frac{8 \pi G V_{0}}{\gamma \cdot(3 \gamma-1)}} \cdot t\right\} \\
& \Leftrightarrow V \approx V_{0} \cdot \exp \left\{-\sqrt{\frac{16 \pi G}{\gamma}} \cdot \phi(t)\right\}
\end{aligned}
$$

Using $r=t \cdot c$

$$
r=t \cdot c=\sqrt{\frac{c^{2} \cdot(3 \gamma-1)}{8 \pi G V_{0}}} \cdot \exp \left(\sqrt{\frac{4 \pi G}{\gamma}} \cdot \phi\right)
$$

Hence, we get a kinetic energy value of

$$
\text { K.E. }=2 m \cdot \frac{\pi G}{\gamma} \cdot\left(\frac{\mathrm{d} \phi}{\mathrm{d} t}\right)^{2} \cdot r^{2}
$$

Thereby leading to

$$
\delta t=\sqrt{\frac{\sum_{i=1}^{N} m_{i} \cdot(\delta d)_{i}^{2}}{2 \cdot(E-V)}}=\sqrt{\frac{\sum_{i=1}^{N} m_{i} \cdot(\delta d)_{i}^{2}}{\sum_{i=1}^{N} m_{i} \cdot\left(\frac{r_{i}}{t}\right)^{2} \cdot\left(\frac{\gamma}{\pi G}\right)}}=\sqrt{\frac{\sum_{i=1}^{N} m_{i} \cdot(\delta d)_{i}^{2}}{\sum_{i=1}^{N} m_{i} \cdot\left(v_{i}\right)^{2} \cdot\left(\frac{\gamma}{\pi G}\right)}}
$$

Here is where we will use the reduced speed of the massive graviton. [9] gives us

$$
v_{\text {graviton }}=c \cdot\left(1-\frac{m_{g}^{2} c^{4}}{E^{2}}\right)^{1 / 2}
$$


Secondly set the $m_{i} \underset{\text { early-universe }}{\longrightarrow} m_{g}$, in the early universe, with $\mathrm{N}$ the number of gravitons.

If we make the following approximation, i.e. $\delta t=10^{x} t_{P}$

$$
E^{2}=\frac{m_{g}^{2} c^{4}}{\left(1-\frac{\pi G}{N \cdot c^{2} \cdot \gamma \cdot\left(10^{\chi} t_{p}\right)^{2}} \cdot \sum_{i=1}^{N}\left(\delta d_{i}\right)^{2}\right)}
$$

This is the net energy associated with a graviton and we will spend the rest of our article analyzing the consequences of such for our questions as what is to known as the $V D V Z$ discontinuity. And its possible resolution.

\section{The Possible Values of $\sum_{i=1}^{N}\left(\delta d_{i}\right)^{2}$ in the Early Universe, as}

\section{Far as the Distribution of N Gravitons}

To do this we will examine what if we are working with a randomized set of value for the $\delta d_{i}$ graviton positions, i.e. roughly like

$$
\delta d_{i} \underset{\text { thermal-cavity }}{\longrightarrow}\left\langle d_{i}\right\rangle_{\text {semi-classical }}
$$

In the case of black body radiation, this would be for a random distribution of "gravitons" in a closed thermal box.

If true, i.e. that assumption. We would likely then be able to generate some version of Bose-Einstein statistics, here, for a graviton "gas" i.e. along the lines of $\mathrm{N}$ for the number of $\mathrm{n}$. assumed gravitons, roughly

$$
N \propto \frac{1}{\left(\exp \left[\frac{E}{k_{B} T}\right]-1\right)}
$$

In this case, we would be revisiting the Solvay conference arguments as of 1927 with respect to [13] [14] [15] [16] [17]. Note that a variant of Equation (9) has also been approved by Weinberg [18]

$$
n(\omega) \mathrm{d} \omega=\frac{\omega^{2} \mathrm{~d} \omega}{\pi^{2}} \cdot\left[\exp \left(\frac{2 \cdot \pi \cdot \hbar \cdot \omega}{\bar{k} T}\right)-1\right]^{-1}
$$

Note that both [19] and [20] in different ways, mean that the neat blackbody radiation approximation assumed in Equation (10) would need huge re adjustments. I.e. [19] would if a model of filament or structural turbulence leading to non-uniform in space-time graviton production, whereas [20] pretty explicitly rules out the idea of a blackbody cavity as far as containment of gravitons.

Hence, we will have to, if either [19] or [20] hold, consider something other than the traditional quantum thermal excitation of say even gravitons within axion walls [21], as has been thought of as possible by this author, and which then may lead to the author positing ways to come up with cosmological dynamics for entries of the terms $\delta d_{i}$ in Equation (7). 
To do that, we will consider, racetrack inflation, and also some of the ideas of what Crowell wrote up in [12]. For entries of the terms $\delta d_{i}$ in Equation (7).

Afterwards, in making some assumptions, as to this set of entries into terms $\delta d_{i}$ in Equation (7) we will go to what we mentioned earlier, which is how to recast the problem of massive gravity in a way which may avoid the $v D V Z$ discontinuity. [3] [4], which will require a long discussion of its own. I.e. mind you this is not meant to be a complete resolution of that problem, but an indication of what our formulation of E, energy, portends to.

\section{First Review of the Racetrack Inflation Scenario and $\sum_{i=1}^{N}\left(\delta d_{i}\right)^{2}$}

This requires looking at Appendix A. And to comment upon what Appendix A has to say about positioning of the space-time domain of production of gravitons, in terms of space-time physics. [22] posits re-heating of the universe, as to where Race-track inflation says the start of graviton production starts.

Quote: From [23]

Once the slow-roll conditions break down, the scalar field switches from being overdamped to being underdamped and begins to move rapidly on the Hubble timescale, oscillating at the bottom of the potential. As it does so, it decays into conventional matter.

End of quote

I.e. this is well after the onset of inflation. [24] indicates that there is a detail of the spectrum which is significant in the initial phases of inflation, as given in [25] [26] [27], and which is given a spectrum value as stated in [24] as

$$
h\left(k, \eta_{i}\right)=A \cdot\left(\frac{k}{k_{H}}\right)^{2+\beta} \operatorname{coth}\left[\frac{k}{2 T}\right]
$$

Specifically, if $\left[\frac{k}{2 T}\right]$ is less than one, due to elevated temperatures, which is what occurs in inflation. Hence, as by [24] the relic condition for gravitational waves cannot be ignored, and [24] states that there is a thermal vacuum state which is given as

$$
\left\langle a_{k}^{\dagger} a_{k^{\prime}}\right\rangle=\left(\frac{1}{\exp (k / T)-1}\right) \cdot \delta^{3}\left(k-k^{\prime}\right)
$$

Notwithstanding what was said about [22] and [23], which appears to rule out significant contributions to relic gravitational waves, due to racetrack we will focus upon what could lead to a thermal vacuum state via racetrack, with comments.

In [28], on page 2 of the article

Quote

Hubble scale during inflation is bounded by the present value of the gravitino mass, i.e., $H<m_{3 / 2}$. This relation, which ties the amplitude of primordial gravi- 
tational waves to the scale of supersymmetry breaking, appears to be rather generic.

End of quote

What this says, is that the racetrack though, in common with other string theory cosmology, has, at the point of symmetry breaking, of the racetrack, a regime where gravitinos, when produced, are giving bounding behavior to the Hubble scale, which in turn [29] [30]

$$
\begin{aligned}
& H^{2} \sim V_{B} / 3 \sim\left|V_{\text {Ads }}\right| / 3 \sim m_{3 / 2}^{2} \\
& \& m_{3 / 2} \sim 6 \times 10^{10} \mathrm{GeV} \\
& \& V_{B} \propto V_{\text {racetrack }}
\end{aligned}
$$

The mass of a graviton, massive, is of the order of [31], so then we use the following [32] [33]

$$
\begin{aligned}
& m_{g} \sim 7.7 \times 10^{-32} \mathrm{GeV} / \mathrm{c} \\
& m_{3 / 2} \sim 6 \times 10^{10} \mathrm{GeV} / \mathrm{c} \sim 10^{42} \mathrm{~m}_{g} \\
& \Rightarrow \text { Electro-weak } \\
& \Rightarrow t_{\text {electro-weak }} \sim 10^{-36} \mathrm{~s} \sim 10^{7} t_{P} \\
& \Rightarrow N \sim 10^{42}
\end{aligned}
$$

Using this, we would have a numerical factor of $\mathrm{N}$, and a time factor of $\delta t$ put in Equation (7)

$$
\begin{aligned}
& \delta t \propto t_{\text {electro-weak }} \sim 10^{-36} \mathrm{~s} \sim 10^{7} t_{P} \\
& N \sim 10^{42}
\end{aligned}
$$

Due to the uncertainty of the exact commencement of the relative distance of the radii of the universe in the electroweak era, [34], we will say then that this relationship will have to be speculated on, in the next section. And this will also incorporate comments on [35]

$$
\delta d_{i} \underset{\text { thermal-cavity }}{\longrightarrow}\left\langle d_{i}\right\rangle_{\text {semi-classical }}
$$

\section{Estimating a Range of Values for $\delta d_{i}$ in Cosmology up to the Electro Weak Era Using Electro Weak Era as the Hot Spot for Relic Graviton Production}

An e fold of 65 in inflation [36] is $10^{28}$ magnification of an initial radius, and so if we consider an electro weak magnification at the end of inflation, for a radii of $10^{-35}$ meters start to a magnified initial radius of about 1 meter at the very end of inflation, tops, with an initial radii of say $10^{-7}$ meters at the start of the electro weak era, to about 1 meter at the close of the electro weak era.

Meaning $N \sim 10^{42}$ gravitons, in a spatial regime of say a ring in between a distance of $10^{-7}$ meters to 1 meter from the 'center' of inflation in a time regime of roughly $\delta t \propto t_{\text {electro-weak }} \sim 10^{-36} \mathrm{~s} \sim 10^{7} t_{P}$.

This would be, if we use the idea of racetrack inflation, and of 1 gravitino roughly equivalent to $N \sim 10^{42}$ gravitons, input into Equation (7).

Keep in mind, that Guth, on page 135, of [37] estimates that the probable total 
reach of inflation is an expansion of up to or more than $10^{75}$ in volume for inflation, i.e. this is then giving us the following inputs, put into Equation (7)

$$
\begin{aligned}
& \delta t \propto t_{\text {electro-weak }} \sim 10^{-36} \mathrm{~s} \sim 10^{7} t_{P} \\
& N \sim 10^{42} \\
& 10^{-7} \text { meters }<\delta d_{i}<1 \text { meter }
\end{aligned}
$$

For the sake of convenience, in this first approximation model we will be initially assuming the rest mass of a graviton is about $10^{-65}$ grams, in line with [31]

We next will, if we assume that there is a correlation between entropy, due to $S$ - $N$ with the number $N=$ (count of particles) [38] next comment upon what this may be saying about entropy, in the early universe.

\section{Review of Different Models of Entropy to Choose from}

This is reviewing the substance of Appendix B and Appendix C below. Here are some first impressions. The given models, do not answer the question of if there is at least one unit of Entropy at the start of the inflationary era. To do that, one can look at what the author did in [39], i.e. the claim is that if one wants to have at least 1 unit of entropy, to start off with, one will have to state a non-zero initial radii. In fact, the author, in [40] generalized this finding via the device of examining a Hubble parameter $=0$, with a jump up to a very large Hubble parameter, right afterwards, as a model for cosmological expansion. I.e. using a cosmological bubble, initially, with $H=0$ at the surface of the bubble, and exploding to the inflation state right afterwards. The idea is materially not different from the phantom bounce, given by Freeze, et al. in [41] as a modus operandi of investigation.

If there are no units of entropy, at the start of expansion of space time, we will choose the methodology of the Racetrack which implies that entropy production and graviton production, and gravity waves would have to await at a minimum, going to the electro weak regime of space time, I.e. That space time expands 10 million times past an initial starting point.

I.e. both the semi classical picture and brane picture tend to support the idea of graviton production starting at the electro-weak era, but if the graviton is a carrier of entropy, and if the radii of the initial configuration of the universe, is not zero, then we will be reason to bring up some of the issues the author raised in [42], which then leads to, if [42] is not wrong, leading to the idea of non-zero initial energy, perhaps recycled from a prior universe, as a starting point for our cosmology. This has implicitly raised the issue of [43], i.e. if there is a $H=0$ initial starting point, of a possible reflection of this, as a causal barrier which may have CMBR overtones.

Note that Beckwith, in [41], generalized a version of the Penrose cyclic conformal cosmology, to multiverses, which may be a way to ascertain if there is, as mentioned earlier, a recycling of space-time, at the start of the universe. In doing so, the author states that this necessitates either a proof, or a counter example to what is given in the traditional [45] with Penrose's supposition as to if there is a 
mandatory singularity at the start of cosmological expansion, as the supposition to either prove or disprove, and with [46] as the non linear electrodynamics speculation to either confirm or falsify as well.

[47] has a lucid competing theory as to non-zero initial radii of the universe speculations, but again, if that is not your favorite, you can peruse the idea brought up, as to using what is given in Crowell, 2005 [12] as to worm hole physics. As asked by the referee, what guarantee that one could use a worm hole as a start to cosmology? Go then to Appendix D, as a start to our discussion. I.e. this is to ascertain if we can say something cogent as to the scale of gravity effects, as either classical or quantum, and then afterwards, go to Appendix E, as to the Crowell-Beckwith suppositions as to worm holes, and the early universe. This is briefly alluded to in Appendix E, which fills in some details as to

$$
\Psi(T) \propto-A \cdot\left\{\eta^{2} \cdot C_{1}\right\}+A \cdot \eta \cdot \omega^{2} \cdot C_{2}
$$

Appendix F, gives a statement largely based upon Mukhanov [48], i.e. how an energy flux from a prior universe may lead to release of entropy in the present universe, and it is in sync with the idea of graviton generation, of early universe entropy after traversing the $\mathrm{H}=0$ barrier in line with the work done in [42] [43] [44].

Appendix $\mathrm{G}$ and Appendix $\mathrm{H}$ give qualitative descriptions as to the behavior of the scalar field, presumably like an inflaton, which may be zero in the initial phases of entry into the "bubble" before a presumed causal barrier at $\mathrm{H}=0$, and Appendix G gives an interpretation of the largeness of a presumed energy flux which would go out of the cosmological "bubble" of initial space time.

Note that the end effect of all this is to argue for very different dynamics, of space time, i.e. for the entropy being generated just past the $\mathrm{H}=0$ barrier of space time, with a radii of say $10^{-35}$ meters, and all that, the answers we will get out of Equation (7) will look profoundly different than say, entropy and gravitons, and GW produced at $10^{-7}$ meters to 1 meter in radii "distance" from the start of presumed space-time.

Either choice will have profound implications for interpreting Equation (7) of our text. What is given below is for what we would have for Equation (7) inputs if we have entropy produced well "before" the electro weak regime

$$
\begin{aligned}
& 10^{-35} \text { meters }<\delta d_{i}<10^{-33} \text { meters } \\
& \& \delta t \propto t_{P} \\
& N \sim 1-10^{10}
\end{aligned}
$$

\section{Conclusion, Now Back to Our Treatment of the Bending of Light by Massive Gravity. What We Can Say about What We Have So Far}

In [49], in Equation (12) of [49], there is an expressed equation of the form for a light ray hitting, say the Sun

$$
\theta \approx \frac{4 G M}{b}+\frac{15 G^{2} M^{2} \pi}{4 b^{2}}+\vartheta\left(\frac{G \hbar M}{b^{3}}=\frac{l_{p}^{2} r_{s}}{b^{3}}\right)
$$


The impact parameter, b of the "photons", i.e. light ray, with the sun, and the Schwartzshield radius $r_{s}$ of the sun.

This is the first item to discuss, and the last term is the one which should be minimized, whereas the first two terms are in sync with [50] [51] [52] whereas the third term, which can be written up in exact parameterization, is too small to contribute much of anything to the problem. I.e. the $2^{\text {nd }}$ term is a post Newtonian contribution and the third term is a quantum correction largely based upon the Born approximation and can be seen in [50], Chapter 21 of that reference. This derivation is part of a manuscript with the following deviation of the potential system put in, i.e.

$$
V=-\frac{G M m}{r} \cdot\left(1+\frac{3 G \cdot(M+m)}{c^{2} r}+\frac{41 G \hbar}{10 c^{3} r^{2}}\right)
$$

If $\mathrm{m}$ is the mass of a graviton, almost $10^{-65}$ grams, whereas $M$ is the mass of a planet, say Mercury, and that Equation (21) has a quantum correction to the tune of $\frac{41 G \hbar}{10 c^{3} r^{2}}$ put in, so that

$$
\begin{aligned}
& V_{\text {quantum-correction }} \approx-\frac{G M m}{r} \cdot\left(\frac{41 G \hbar}{10 c^{3} r^{2}}\right) \\
& \Leftrightarrow \theta_{\text {quantum-correction }} \approx \vartheta\left(\frac{G \hbar M}{b^{3}}=\frac{l_{p}^{2} r_{s}}{b^{3}}\right) \propto \varepsilon^{+} \approx 0
\end{aligned}
$$

Our task would be to look at a total energy, say making this deduction, of

$$
\begin{aligned}
& E_{\text {graviton }}^{2}=\frac{m_{g}^{2} c^{4}}{\left(1-\frac{\pi G}{N \cdot c^{2} \cdot \gamma \cdot\left(10^{\chi} t_{P}\right)^{2}} \cdot \sum_{i=1}^{N}\left(\delta d_{i}\right)^{2}\right)} \\
& \Leftrightarrow V_{\text {graviton-Sun }}=-\frac{G M m}{r} \cdot\left(1+\frac{3 G \cdot(M+m)}{c^{2} r}+\frac{41 G \hbar}{10 c^{3} r^{2}}\right) \\
& \Leftrightarrow V_{\text {quantum-correction }} \approx-\frac{G M m}{r} \cdot\left(\frac{41 G \hbar}{10 c^{3} r^{2}}\right) \\
& \Leftrightarrow \theta_{\text {quantum-correction }} \approx \vartheta\left(\frac{G \hbar M}{b^{3}}=\frac{l_{p}^{2} r_{s}}{b^{3}}\right) \propto \varepsilon^{+} \approx 0
\end{aligned}
$$

It would be a lot of work, but it would also be more direct than what De Rahm and other tried in [53].

What we have done, is to find a basis for a different way to address the issue of if we have relic gravitational waves at just the electro weak regime, as quantified in this paper, or if we have earlier based processes and/or the influence of recycled earlier universes, which may influence the transmission of gravitons, and possibly pre universe information to our present universe.

Do we have a repeating universe, with shared from the prior cosmos information? The logical extension of the inquiry so presented may allow for answering this question. In the meantime, the touch of using Barbour's version of time, in- 
itially was put in to ascertain, a working benchmark for the twinning of a definite time step, with graviton production, and also then, if graviton production, i.e. the number of gravitons, is proportional to entropy, what has been done is in essence vetting the start of times arrow, via entropy production in the universe.

Equation (7) is by necessity very preliminary and we expect to revisit it with greater precision later on.

Finally we have presented a different way to start an inquiry as to working to a solution to the $v D V Z$ discontinuity.

See Appendix I, as to the remarks made as to the foundations of gravitational astronomy. The document so presented is expected to be in fidelity with respect to these observations and guidelines.

\section{Acknowledgements}

This work is supported in part by National Nature Science Foundation of China grant No. 11375279.

\section{References}

[1] Fierz, M. and Pauli, W. (1939) On Relativistic Wave Equations for Particles of Arbitrary Spin in an Electromagnetic Field. Proceedings of the Royal Society of London $A, 173,211-232$. https://doi.org/10.1098/rspa.1939.0140

[2] van Dam, H., Veltman, M. (1970) Massive and Massless Yang-Mills and Gravitational Fields. Nuclear Physics B, 22, 397-411.

https://doi.org/10.1016/0550-3213(70)90416-5

[3] Zakharov, V.I. (1970) Linearized Gravitation Theory and the Graviton Mass. JETP Letters, 12, 312.

[4] Vainshtein, A.I. (1972) To the Problem of Nonvanishing Gravitation Mass. Physics Letters B, 39, 393-394. https://doi.org/10.1016/0370-2693(72)90147-5

[5] Maggiore, M. (2008) Gravitational Waves, Volume 1, Theory and Experiments. Oxford University Press, Oxford.

[6] Barbour, J. (2009) The Nature of Time. https://arxiv.org/abs/0903.3489

[7] Anderson, E. (2004) Geometrodynamics: Spacetime or Space? arXiv:gr-qc/0409123 [gr-qc]

[8] Butterfield, J. (1999) The Arguments of Time. Oxford University Press, Oxford.

[9] Will, C. (2014) The Confrontation between General Relativity and Experiment. Living Reviews in Relativity, 17, 4.

https://arxiv.org/abs/1403.7377 https://doi.org/10.12942/lrr-2014-4

[10] Padmanabhan, T. (2006) An Invitation to Astrophysics. World Scientific, Singapore.

[11] Blanco-Pillado, J.J., Burgess, C.P., Cline, J.M., Escoda, C., Gomez-Reino, M., Kallosh, R., Linde, A. and Quevedo, F. (2004) Racetrack Inflation. Journal of High Energy Physics, 2004, JHEP11. https://arxiv.org/abs/hep-th/0406230 https://doi.org/10.1088/1126-6708/2004/11/063

[12] Crowell, L. (2005) Quantum Fluctuations of Space Time. World Scientific Series in 
Contemporary Chemical Physics, 25, 388 p. https://doi.org/10.1142/5952

[13] Omnes, R. (1999) Understanding Quantum Mechanics. Princeton University Press, Princeton, New Jersey.

[14] Omnes, R. (1994) The Interpretation of Quantum Mechanics. Princeton University Press, Princeton, New Jersey.

[15] Bacciagaluppi, G. and Valentini, A. (2009) Quantum Theory at the Crossroads: Reconsidering the 1927 Solvay Conference. Cambridge University Press, Cambridge, Massachusetts. https://doi.org/10.1017/CBO9781139194983

[16] Gasiorowitz, S. (2003) Quantum Physics. 3rd Edition, Wiley Interscience, Hoboken, New Jersey.

[17] Mehra, J. and Rechenberg, G. (1982) The Historical Development of Quantum Theory, Volume 4, Part 1 The Fundamental Equations of Quantum Mechanics, 1925-1926, and Part 2 The Reception of the New Quantum Mechanics, 1925-1926. Springer-Verlag, New York.

[18] Weinberg, S. (1972) Gravitation and Cosmology: Principles and Applications of the General Theory of Relativity. John Wiley \& Sons, Inc., New York.

[19] Ruutu, V., Eltsov, V., Gill, A., Kibble, T., Krusius, M., Makhlin, Y.G., Placais, B., Volvik, G. and Wen, Z. (1996) Vortex Formation in Neutron-Irradiated ${ }^{3} \mathrm{He}$ as an Analog of Cosmological Defect Formation. Nature, 382, 334-336. https://doi.org/10.1038/382334a0

[20] Enqvist, K., Mazumdar, A. and Perez-Lorenzana, A. (2004) Dumping Inflaton Energy Out of This World. Physical Review D, 70, Article ID: 103508. https://doi.org/10.1103/PhysRevD.70.103508

[21] Beckwith, A.W. (2006) Does A Randall-Sundrum Brane World Effective Potential Influence Axion Walls Helping to Form a Cosmological Constant Affecting Inflation? Journal of High Energy Physics, Gravitation and Cosmology, 2, 125-153. http://dx.doi.org/10.4236/jhepgc.2016.21013

[22] Kofman, L. and Yi, P. (2005) Reheating the Universe after String Theory Inflation. https://arxiv.org/pdf/hep-th/0507257.pdf

[23] https://ned.ipac.caltech.edu/level5/Liddle/Liddle5_6.html

[24] Ghayour, B. and Khodagholizadeh, J. (2017) Detection of Relic Gravitational Waves in Thermal Case by Using Adv.LIGO Data of GW150914. The European Physical Journal C, 77, 560. https://doi.org/10.1140/epjc/s10052-017-5135-8

[25] Zhao, W., et al. (2009) Detecting Relics of A Thermal Gravitational Wave Background in the Early Universe. Physics Letters B, 680, 411-416. https://doi.org/10.1016/j.physletb.2009.09.018

[26] Ghosh, T.K., Pal, S., Sinha, T., Majumdar, N., Chattopadhyay, S., Bhattacharya, P., et al. (2004) Anomalous Increase in Width of Fission Fragment Mass Distribution as a Probe for Onset of Quasifission Reactions in Deformed Target-Projectile System at Near and Sub-Barrier Energies. Physical Review C, 70, 011604(R).

[27] Wang, K., Santos, L., Xia, J.Q. and Zhao, W. (2017) Thermal Gravitational Wavebackground in the General Pre-Inflationary Scenario. arXiv:1608.04189 [astro-ph.CO]

[28] Chen, H.-Y., Hung, L.-Y. and Shiu, G. (2009) Inflation on an Open Racetrack. https://arxiv.org/pdf/0901.0267.pdf

[29] Kallosh, R. and Linde, A. (2004) Landscape, the Scale of SUSY Breaking, and Inflation. JHEP 0412, 004. [arXiv:hep-th/0411011]. 
[30] Kachru, S., Kallosh, R., Linde, A. and Trivedi, S.P. (2003) De Sitter Vacua in String Theory. Physical Review D, 68, Article ID: 046005. https://doi.org/10.2172/812628

[31] Abbott, B.P., et al. (2017) LIGO Scientific Collaboration and Virgo Collaboration. GW170104: Observation of a 50-Solar-Mass Binary Black Hole Coalescence at Redshift 0.2. Physical Review Letters, 118, Article ID: 221101. https://doi.org/10.1103/PhysRevLett.118.221101

[32] Ryden, B. (2003) Introduction to Cosmology. Addison-Wesley, Boston, Massachusetts, 196.

[33] Allday, J. (2002). Quarks, Leptons and the Big Bang. Taylor \& Francis, London, 334.

[34] Romão, J.C. and Freire, F. (1994) Electroweak Physics and the Early Universe. North Atlantic Treaty Organization, Scientific Affairs Division Plenum Press, Science, 410 p. https://doi.org/10.1007/978-1-4899-1304-3

[35] Coleman, S. and De Luccia, F. (1980) Gravitational Effects on and of Vacuum Decay. Physical Review D, D21, 3305-3315. https://doi.org/10.1103/PhysRevD.21.3305

[36] Freeze, K. (1992) Inflation. In: Nath, P. and Reucroft, S., Eds., Particles, Strings, and Cosmology, World Scientific, Singapore, 408-424.

[37] Guth, A. (1989) Starting the Universe: The Big Bang and Cosmic Inflation. In: Cornell, J., Ed., Bubbles, Voids and Bumps in Time: The New Cosmology, Cambridge University Press, New York, 105-146.

[38] Ng, Y.J. (2008) Spacetime Foam: From Entropy and Holography to Infinite Statistics and Nonlocality. Entropy, 10, 441-461. https://doi.org/10.3390/e10040441

[39] Beckwith, A. (2017) Isolating a Minimum Radius of the Universe Consistent with the Production of at Least 1 Unit of Entropy, at the Start of Inflation. Journal of High Energy Physics, Gravitation and Cosmology, 3, 461-466. https://doi.org/10.4236/jhepgc.2017.33036

[40] Beckwith, A. (2017) How to Determine Initial Starting Time Step with an Initial Hubble Parameter $\mathrm{H}=0$ after Formation of Causal Structure Leading to Investigation of the Penrose Weyl Tensor Conjecture. http://vixra.org/abs/1706.0110

[41] Freeze, K., Brown, M. and Kinney, W. (2012) The Phantom Bounce: A New Proposal for an Oscillating Cosmology. In: Mersini-Houghton, L. and Vaas, R., Eds., The Arrows of Time, A Debate in Cosmology, Springer Verlag, Heidelberg, 149-156. https://doi.org/10.1007/978-3-642-23259-6_7

[42] Beckwith, A. (2017) How to Determine a Jump in Energy Prior to a Causal Barrier, with an Attendant Current, for an Effective Initial Magnetic Field. in the Pre Planckian to Planckian Space-Time. http://vixra.org/abs/1707.0250

[43] Beckwith, A. (2016) Open Question: Could a Causal Discontinuity Explain Fluctuations in the CMBR Radiation Spectrum? Journal of High Energy Physics, Gravitation and Cosmology, 2, 186-208. https://doi.org/10.4236/jhepgc.2016.22018

[44] Beckwith, A.W. (2014) Analyzing Black Hole Super-Radiance Emission of Particles/Energy from a Black Hole as a Gedanken Experiment to Get Bounds on the Mass of a Graviton. Hindawi Publishing Corporation Advances in High Energy Physics, 2014, Article ID: 230713, 7 p.

[45] Penrose, R. (1983) Spinors and Torsion in General Relativity. Foundations of Physics, 13, 325-339. https://doi.org/10.1007/BF01906181

[46] Camara, C.S., de Garcia Maia, M.R., Carvalho, J.C. and Lima, J.A.S. (2004) Nonsingular FRW Cosmology and Non Linear Dynamics. Arxiv astro-ph/0402311

[47] Rovelli, C., and Vidotto, F. (2015) Covariant Loop Quantum Gravity, an Elementary 
Introduction to Quantum Gravity, and Spinfoam Theory. Cambridge University Press, Cambridge.

[48] Mukhanov, V. (2005) Physical Foundations of Cosmology. Cambridge University Press, Cambridge. https://doi.org/10.1017/CBO9780511790553

[49] Bjerrum-Bohr, N.E.J., Donoghue, J.F., Holstein, B.R., Planté, L. and Vanhove, P. (2015) Bending of Light in Quantum Gravity. Physical Review Letters, 114, Article ID: 061301. https://doi.org/10.1103/PhysRevLett.114.061301

[50] Bohm, D. (1989) Quantum Theory. Dover, New York.

[51] Donoghue, J.F. and Holstein, B.R. (1986) Quantum Mechanics in Curved Space. American Journal of Physics, 54, 827. https://doi.org/10.1119/1.14423

[52] Bodenner, J. and Will, C.M. (2003) Deflection of Light to Second Order: A Tool for Illustrating Principles of General Relativity. American Journal of Physics, 71, 770. https://doi.org/10.1119/1.1570416

[53] de Rham, C., Tolley, A.J. and Zhou, S.-Y. (2016) The $\Lambda 2$ Limit of Massive Gravity. https://arxiv.org/pdf/1602.03721.pdf

[54] Brax, Ph., Davis, A., Davis, S., Jeannerot, R. and Postman, M. (2008) D-Term Uplifted Racetrack Inflation. Journal of Cosmology and Astroparticle Physics, 2008.

[55] Hunt, P. and Sarkar, S. (2007) Multiple Inflation and the WMAP “Glitches”. II. Data Analysis and Cosmological Parameter Extraction. Physical Review D, 76, Article ID: 123504. https://doi.org/10.1103/PhysRevD.76.123504

[56] Kolb, E. and Turner, S. (1994) The Early Universe. Westview Press, Chicago.

[57] Maggiore, M. (2008) Gravitational Waves, Volume 1: Theory and Practice. Oxford University Press, Oxford.

[58] Lloyd, S. (2002) Computational Capacity of the Universe. Physical Review Letters, 88, Article ID: 237901. https://doi.org/10.1103/PhysRevLett.88.237901

[59] Padmanabhan, T. (2000) Theoretical Astro Physics, Volume 1: Astrophysical Processes. Cambridge University Press, Cambridge.

[60] Mathur, S. and Chowdhury, B. (2007) Fractional Brane States in the Early Universe. Classical and Quantum Gravity, 24, 2689-2720. https://doi.org/10.1088/0264-9381/24/10/014

[61] Lifschytz, G. (2004) Black Hole Thermalization Rate from Brane Anti Brane Model. Journal of High Energy Physics, 2004, JHEP08. https://doi.org/10.1088/1126-6708/2004/08/059

[62] Park, D.K., Kim, H. and Tamarayan, S. (2002) Nonvanishing Cosmological Constant of Flat Universe in Brane World Senarios. Physics Letters B, 535, 5-10. https://doi.org/10.1016/S0370-2693(02)01729-X

[63] Padmanabhan, T. (2005) Understanding Our Universe: Current Status and Open Issues. In: Ashtekar, A., Ed., 100 Years of Relativity Space-Time Structure: Einstein and Beyond, World Press Scientific, Singapore.

[64] Padmanabhan, T. (2006) An Invitation to Astrophysics. World Press Scientific, Singapore.

[65] Abbott, B.P., et al. (2016) Observation of Gravitational Waves from a Binary Black Hole Merger. $P R L, 116$, Article ID: 061102. https://doi.org/10.1103/PhysRevLett.116.061102

[66] Corda, C. (2009) Interferometric Detection of Gravitational Waves: The Definitive Test for General Relativity. International Journal of Modern Physics D, 18, 2275-2282. https://doi.org/10.1142/S0218271809015904 
[67] Abbot, B.P., et al. (2016) GW151226: Observation of Gravitational Waves from a 22-Solar-Mass Binary Black Hole Coalescence. Physical Review Letters, 116, Article ID: 241103. https://doi.org/10.1103/PhysRevLett.116.241103

[68] Halliwell, J. (1991) Quantum Cosmology. In: Pati, J.R.-D., Sezgin, E. and Shafi, Q., Eds., The ICTP Serioes in Theoretical Physics, Word Scientific, Singapore, 515 -599,

[69] Beckwith, A. (2017) Is Temperature Quenching in the Early Universe Due to Particle Production, or Quantum Occupation States, or the Influence of Quantum Teleportation? http://vixra.org/abs/1701.0545?ref=9544533

[70] Beckwith, A. (2017) Showing Fjortof's Theorem Does Not Apply for Defining Instability for Early Universe Thermodynamic Potentials. Asking if Nucleated Particles Result At/before Electro-Weak Era Due to Injection of Matter-Energy at the Big Bang? http://vixra.org/abs/1709.0017?ref=9543034 


\section{Appendix A: Managing What to Do with Racetrack Inflation, as Cool down from Initial Expansion Commences}

P. Brax, A. Davis et al. [54] devised a way to describe racetrack inflation as a way to look at how super gravity directly simplifies implementing how one can have inflation with only three $\mathrm{T}$ (scalar) fields. The benefit to what we work with is that we may obtain two gaugino condensates and look at inflation with a potential given by [54]

$$
V=V_{0}+V_{1} \cos (a Y)+V_{2} \cos (b Y)+V_{3} \cos (|a-b| \cdot Y)
$$

This has scalar fields $X, \phi$ as relatively constant and we can look at an effective kinetic energy term along the lines of

$$
\mathfrak{\Im}_{\text {Kinetic }}=3 \cdot(\partial Y)^{2} / 4(\partial X)^{2}
$$

This ultra simple version of the race track potential is chosen so that the following conditions may be applied

1) Exist a minimum at $Y=Y_{0}$; i.e. we have $V^{\prime}\left(Y_{0}\right)=0$, and $V^{\prime}\left(Y_{0}\right)>0$, when we are not considering scalar fields $X, \phi$

2) We set a cosmological constant equal to zero with $V\left(Y_{0}\right)=0$

3) We have a flat saddle at $Y \approx 0$; I.e. $V^{\prime \prime}(0)=0$

4) We re-scale the potential via $V \rightarrow \lambda V$ so as to get the observed power spectra $P=4 \times 10^{-10}$

Doing all this though frequently leads to the odd situation that $|a-b|$ must be small so that $X \gg 1$ in a race track potential system when we analyze how to fit Equation (1) for flat potential behavior modeling inflation. This assumes that we are working with a spectra index of the form so that if the scalar field power spectrum is

$$
P=\frac{V}{150 \pi^{2} \varepsilon}
$$

Then the spectral index of the inflaton is consistent with WMAP data. I.e. if we have the number of e foldings $N>N_{*} \approx 0.55$

$$
n_{s}=1-\frac{\mathrm{d} \ln P}{\mathrm{~d} N} \approx 0.95 \pm 0.02
$$

These sort of restrictions on the spectral index will start to help us retrieve information as to possible inflation models which may be congruent with at least one layer of WMAP data. This model says nothing about if or not the model starts to fit in the data issues Subir Sarkar [55] identified in is Pune, India lecture in 2007.

\section{Appendix B: Semi Classical Models of Entropy Generation}

Kolb and Turner [56] have a temperature $T$ related entropy density which can be treated as being written as:

$$
S_{\text {Density }}=\frac{2 \cdot \pi^{2}}{45} \cdot g_{*} \cdot T^{3}
$$


This pre supposes when we do it that we are able to state a total entropy as the entropy density times space time volume $V_{4}$

$$
S_{\text {total }} \equiv S_{\text {Density }} \cdot V_{4}
$$

In this situation we are writing for initial conditions with a temperature $T \approx 10^{32} \mathrm{~K}$ for the initiation of quantum effects for quantum gravity as given by Weinberg (1972) [18] which is further elaborated upon by Padmanabhan, in [57].

$T \approx 10^{32} \mathrm{~K} \approx 1.3 \times 10^{28} \mathrm{eV} \sim 1.3 \times 10^{19} \mathrm{GeV}$. This gives us the option of comparing what we get in entropy with Seth Lloyds [58]

$$
I=S_{\text {total }} / k_{B} \ln 2=[\# \text { operations }]^{3 / 4}=\left[\rho \cdot c^{5} \cdot t^{4} / \hbar\right]^{3 / 4}
$$

We will examine if or not the following is actually true in terms of time, i.e. can we write $I=\left(t / t_{P}\right)^{2}$ ? This is assuming that the density

$\rho \equiv T^{00} \sim \Lambda_{\text {vacuum-energy }}$ which is initially enormous, and which will be due in terms of a transfer of energy density from a prior universe to our present universe, which will be elaborated upon later in this document.

We can if we take the absolute value of Equation (b3) and (b2) above get for small volume values good estimates as to the relative volume of the phase space in early universe cosmology where Equation (b2) and Equation (b3) are congruent with each other. For our purposes, we will take time as greater than (or equal) to a Planck time interval, in line with the temperature dependence of entropy density mentioned in Equation (b1) above.

We can compare this with Thanu Padamanadan's [59] treatment of entropy which is with regards to micro canonical ensemble as defined via

$$
\begin{aligned}
\exp \left(S_{\text {total }}\right) & =g(E)=\frac{A}{N !} \cdot \int \mathrm{d}^{3 N} x \cdot\left[E-\frac{1}{2} \cdot \sum_{i \neq j} U\left(x_{i}, x_{j}\right)\right]^{\frac{3 N}{2}} \\
& \leq \frac{A}{N !} \cdot \int \mathrm{d}^{3 N} X \cdot[E]^{\frac{3 N}{2}} \approx\left[\frac{A}{N !} \cdot \int \mathrm{d}^{3 N} X\right] \cdot\left[\frac{\Lambda_{M a x} V_{4}}{8 \cdot \pi \cdot G}\right]^{\frac{3 N}{2}}
\end{aligned}
$$

If $A \sim O(1)$, i.e. we re scale it as being of order unity, and $N \sim 10^{87}$ particles, and we re scale $\int \mathrm{d}^{3 N} x \sim V_{4}^{N}$ where we choose $V_{4}$, and where we assume Equation (b2) and Equation (b3) are equivalent and we assume that there is grounds for writing $\frac{\Lambda_{\mathrm{Max}} V_{4}}{8 \cdot \pi \cdot G} \sim T^{00} V_{4} \equiv \rho \cdot V_{4} \gg \frac{1}{2} \cdot \sum_{i \neq j} U\left(x_{i}, x_{j}\right)$, we can shed light on if or not it is still feasible to treat entropy, with $N \sim 10^{87}$ as a micro canonical ensemble phenomena, which we claim has implications for the formation of an instanton in early universe cosmology. Frankly we would want, in early universe cosmology that we have $\rho \cdot V_{4} \neq \frac{1}{2} \cdot \sum_{i \neq j} U\left(x_{i}, x_{j}\right)$, but not by too much, so we can form an instanton.

\section{Appendix C: Brane World Picture of Early Universe Entropy Formation}

This is adapted from a lecture given at the ICGC-07 conference by Samir Mathur 
[60]. The supposition is that branes and anti branes form the working component of an instanton, which is part of what has been developed.

I.e. look at the case, first of massless radiation, and then we obtain for $\mathrm{D}$ space time dimensions, and $\mathrm{E}$ the general energy

$$
S \sim E^{(D-1 / D)}
$$

This has

$$
\frac{\Lambda_{\mathrm{Max}} V_{4}}{8 \cdot \pi \cdot G} \sim T^{00} V_{4} \equiv \rho \cdot V=E_{\text {total }}
$$

The question now becomes how do we go about defining what the necessary volume is re scaled via a quantum gravity changing of how to measure gravitational lengths which are for the threshold of quantum gravity. Traditionally the bench marking has been via the Planck length

$l_{P} \sim 10^{-33} \mathrm{~cm} \longrightarrow$ Quantum-Gravity-threshold $\longrightarrow \tilde{N}^{\alpha} \cdot l_{P}$. This re scaling of the minimum length needed for the importance of quantum gravity effects showing up in a grid of space time resolves, as information paradox of black hole physics. So far we have merely been working with a typical string gas model for entropy. Now, let us add in a supposition for $\breve{N}$ branes and anti branes to put in an instanton structure as to how we look at the entropy. Gilad Lifschytz [61] in 2004 codified thermalization equations of the black hole which was recovered from the model of branes and anti-branes, and in lieu of assuming an anti brane is merely in this situation the charge conjugate of say a Dp brane wrote an entropy along the lines of modifying Equation (c1) above to read

$$
S_{\text {Total }} \sim \breve{a} \cdot\left[\frac{E_{\text {Total }}}{2^{n}}\right]^{\lambda} \cdot \prod_{j=1}^{N}\left(\sqrt{M_{p j, 0}}+\sqrt{M_{\bar{p} j, 0}}\right)
$$

This has when we do it $E_{\text {Total }}$ as in Equation(c1) above, and proportional to the cosmological vacuum energy parameter. Of course, in string theory, the energy is also defined via

$$
E_{\text {Total }}=4 \lambda \cdot \sqrt{M_{p j, 0} \cdot M_{\bar{p} j, 0}}
$$

Furthermore, the values of $M_{p j, 0}$, and $M_{\bar{p} j, 0}$ refer to the mass of $\mathrm{p}$ branes and $p$ anti branes, as Gilad Lifschytz refers to it. This can be changed and rescaled to treating the mass and the energy of the brane contribution along the lines of Mathur's ${ }^{2}$ [60] CQG article where he has a string winding intepretation of energy along the lines of putting as much energy $E$ into string windings as possible via, $\left[n_{1}+\bar{n}_{1}\right] L T=\left[2 n_{1}\right] L T=E / 2$, where we are talking about $n_{1}$ wrappings of a string about a cycle of the torus, and $\bar{n}_{1}$ being "wrappings the other way", with the torus having a cycle of length $L$, which leads to an entropy defined in terms of an energy value of, if mass $m_{i}=T_{P} \prod L_{j}$ (with $T_{P}$ being the tension of the ith brane, and $L_{j}$ being spatial dimensions of a complex torus structure)

$$
E_{\text {Total }}=2 \sum_{i} m_{i} n_{i}
$$


This leads to entropy

$$
S_{\text {Total }}=A \cdot \prod_{i}^{N} \sqrt{n_{i}}
$$

Our claim is that this very specific value of entropy for Equation (c6) above will in Planck interval of time at about the onset of inflation lead to

$$
\left|\left[S_{\text {Total }}=A \cdot \prod_{i}^{N} \sqrt{n_{i}}\right] / k_{B} \ln 2\right| \approx[\# \text { operations }]^{3 / 4} \approx 10^{8}
$$

Furthermore we also claim that the interaction of the branes and anti-branes will form an instanton structure, which is implicit in the treatment outlined in Equation (c4), and that the numerical counting given in Equation (c6) merely reflects that branes and anti-branes, even if charge conjugates of each other have the same "wrapping number" $n_{i}$.

\section{Appendix D: Specific Numerical Estimates for the Minimum Boundary of Quantum Gravity Volume vs. Classical Gravity Dominated Effects}

We begin with a temperature estimate of $T \approx 10^{45} \mathrm{~K}>T_{\mathrm{QG}-\text { Threshold }} \sim 10^{32} \mathrm{~K}$. Then, Equation (b4) above modified when we take the absolute value will lead to, if we look at when $N \approx 10^{86}$ :

$$
\left|S_{\text {Total }}\right| \sim\left|k_{B} \cdot \ln 2\right| \cdot[\# \text { operations }]^{3 / 4} \approx N\left|-\log \frac{N}{10}+\log V_{4}^{3}+\log E^{3 / 2}\right| \sim 10^{8}
$$

Leading to solving for $\mathrm{E}$ as follows $\Rightarrow \rho \cdot V_{4} \neq \frac{1}{2} \cdot \sum_{i \neq j} U\left(x_{i}, x_{j}\right)$, and also that

$$
V_{4}^{3} \cdot E^{3 / 2} \sim 10^{85} \Rightarrow E \sim \frac{10^{57}}{V_{4}^{2}} \equiv \frac{\Lambda_{\mathrm{Max}} \cdot V_{4}}{8 \cdot \pi \cdot G}
$$

We can and will reference what we can say about $\Lambda_{\text {Max }} \sim C_{2} \cdot T^{\tilde{\beta}}$, as given by Park [62] (2003), as a way to get an upper bound estimate upon $V_{4}$ for quantum gravity effects in inflation. We get an upper bond estimate of

$$
\left.V_{4}\right|_{\text {Threshold-volume-for-quantum-effects }} \sim 10^{-4} \mathrm{~cm}
$$

This is way too large, but it indicates that the interaction of material within the region of space being considered does not obey $\rho \cdot V_{4} \gg \frac{1}{2} \cdot \sum_{i \neq j} U\left(x_{i}, x_{j}\right)$. If this is what we have, we can then begin to look at if the instanton picture is true or not. We will first review what can be said about different variants of vacuum energy. I.e. where the vacuum energy models of four and five dimensions could conceivably overlap. But to do this we will look at what these models are.

\section{Appendix E: Details as to Forming Crowell's Time Dependent Wheeler De Witt Equation, and Its Links to Worm Holes}

We will fill in the details inherent in Equation (18) above in the main text.. This 
will be to show some things about the worm hole we assert the instanton traverses en route to our present universe. Equation (18) of the main text actually comes from the following version of the Wheeler De Witt equation with a pseudo time component added. From Crowell [12]

$$
-\frac{1}{\eta r} \frac{\partial^{2} \Psi}{\partial r^{2}}+\frac{1}{\eta r^{2}} \cdot \frac{\partial \Psi}{\partial r}+r R^{(3)} \Psi=(r \eta \phi-r \ddot{\phi}) \cdot \Psi
$$

This has when we do it $\phi \approx \cos (\omega \cdot t)$, and frequently $R^{(3)} \approx$ constant, so then we can consider

$$
\phi \cong \int_{0}^{\infty} \mathrm{d} \omega\left[a(\omega) \cdot \mathrm{e}^{i k_{\sigma} x^{\mu}}-a^{+}(\omega) \cdot \mathrm{e}^{-i k_{\sigma} x^{\mu}}\right]
$$

In order to do this, we can write out the following with regards to the solutions to Equation (e1) put up above.

$$
\begin{aligned}
C_{1}= & \eta^{2} \cdot\left(4 \cdot \sqrt{\pi} \cdot \frac{t}{2 \omega^{5}} \cdot J_{1}(\omega \cdot r)+\frac{4}{\omega^{5}} \cdot \sin (\omega \cdot r)+(\omega \cdot r) \cdot \cos (\omega \cdot r)\right) \\
& +\frac{15}{\omega^{5}} \cos (\omega \cdot r)-\frac{6}{\omega^{5}} \operatorname{Si}(\omega \cdot r)
\end{aligned}
$$

And

$$
C_{2}=\frac{3}{2 \cdot \omega^{4}} \cdot(1-\cos (\omega \cdot r))-4 \mathrm{e}^{-\omega \cdot r}+\frac{6}{\omega^{4}} \cdot \operatorname{Ci}(\omega \cdot r)
$$

This is where $\operatorname{Si}(\omega \cdot r)$ and $\operatorname{Ci}(\omega \cdot r)$ refer to integrals of the form $\int_{-\infty}^{x} \frac{\sin \left(x^{\prime}\right)}{x^{\prime}} \mathrm{d} x^{\prime}$ and $\int_{-\infty}^{x} \frac{\cos \left(x^{\prime}\right)}{x^{\prime}} \mathrm{d} x^{\prime}$. It so happens that this is for forming the wave functional permitting an instanton forming, while we next should consider if or not the instanton so farmed is stable under evolution of space time leading up to inflation. We argue here that we are forming an instanton whose thermal energy is focused into a wave functional which is in the throat of the worm hole up to a thermal discontinuity barrier at the onset, and beginning of the inflationary era.

\section{Appendix F: Energy Fluctuations Due to the Worm Hole and Their Link to Entropy Fluctuations}

We argue that the existence of the worm hole and an instanton formation in the throat of the worm hole will lead to a constant energy flux. Note that we are assuming a constant energy flux through the worm hole. This is equivalent to work with an expression given by Mukhanov [48] about energy density fluctuations and entropy. In position space, it is for energy density $\rho(x)$, and entropy $S(x)$

$$
\frac{\partial^{2} \delta \rho(x)}{\partial t^{2}}-c_{s}^{2} \Delta \cdot \delta \rho(x)-4 \pi \cdot G \rho_{0} \cdot \delta \rho(x)=\sigma \cdot \Delta \delta S(x)
$$

This is Fourier transformed into being

$$
\frac{\partial^{2} \delta \rho(k)}{\partial t^{2}}+k^{2} c_{s}^{2} \cdot \delta \rho(k)-4 \pi \cdot G \rho_{0} \cdot \delta \rho(k)=-\sigma \cdot k^{2} \delta S(k)
$$


This has a time independent solution of the form given by, assuming small spatial dimensions

$$
\delta \rho(k) \equiv-\frac{\sigma k^{2} \delta S(k)}{\left(k^{2} c_{s}^{2}-4 \pi G \rho_{0}\right)} \underset{k \rightarrow \text { big }}{\longrightarrow}-\frac{\sigma \delta S(k)}{\left(c_{s}^{2}\right)}
$$

This may be Fourier transformed, assuming near constant values of $k$ and position $\mathrm{x}$, to be in $\mathrm{x}$ position space

$$
\delta \rho(x) \cong-\frac{8 \sigma}{c_{s}^{2}} \delta S(x)
$$

Here, $c_{s}^{2}$ is the square of the speed of sound which is in early universe conditions close to unity. We also have that $\sigma \equiv(\partial p / \partial S)_{\rho}$. Then we can state that when we have $\delta \rho(x) \propto \Lambda_{\text {initial }} \rightarrow \Lambda_{\max }$ due to increasing temperature

$$
|\delta \rho(x)| \cong 8 \sigma \cdot \delta S(x)
$$

We claim that the increase in entropy, is connected with a breaking of the instanton structure of a packet of energy transferred from a prior space time to our own.

\section{Appendix G: First Principles Argument as to Large Scale Values of the Absolute Magnitude of the Cosmological Vacuum Energy}

Look at an argument provided by Thanu Padmanabhan [10] [63], leading to the observed cosmological constant value suggested by Park [62]. Assume that $l_{P} \sim 10^{-33} \mathrm{~cm} \underset{\text { Quantum-Gravity-threshold }}{\longrightarrow} \tilde{N}^{\alpha} \cdot l_{P}$, but that when we make this substitution that $1 \leq \tilde{N}^{\alpha} \leq 10^{2} \quad[63]$

$$
\begin{gathered}
\rho_{V A C} \sim \frac{\Lambda_{\text {observed }}}{8 \pi G} \sim \sqrt{\rho_{U V} \cdot \rho_{I R}} \sim \sqrt{l_{\text {Planck }}^{-4} \cdot l_{H}^{-4}} \sim l_{\text {Planck }}^{-2} \cdot H_{\text {observed }}^{2} \\
\Delta \rho \approx \text { a dark energy density } \sim H_{\text {observed }}^{2} / G
\end{gathered}
$$

We can replace $\Lambda_{\text {observed }}, H_{\text {observed }}^{2}$ by $\Lambda_{\text {initial }}, H_{\text {initial }}^{2}$. In addition we may look at inputs from the initial value of the Hubble parameter to get the necessary e folding needed for inflation, according to

$$
\begin{aligned}
& E \text {-foldings }=H_{\text {initial }} \cdot\left(t_{\text {End of inf }}-t_{\text {beginning of inf }}\right) \equiv N \geq 100 \\
& \Rightarrow H_{\text {initial }} \geq 10^{39}-10^{43}
\end{aligned}
$$

Leading to

$$
a(\text { End of inf }) / a(\text { Beginning of inf }) \equiv \exp (N)
$$

If we set $\Lambda_{\text {initial }} \sim C_{1} \cdot\left[T \sim 10^{32}\right.$ Kelvin $]$ implying a very large initial cosmological constant value, we get in line with what Park [62] suggested for times much less than the Planck interval of time at the instant

of nucleation of a vacuum state

$$
\Lambda_{\text {initial }} \sim\left[10^{156}\right] \cdot 8 \pi G \approx \text { huge number }
$$


Question. Do we always have this value of Equation (g5)? At the onset of Inflation? When we are not that far away from a volume of space characterized by $l_{P}^{3}$, or at most 100 or so times larger? Contemporary big bang theories imply this. I.e. a very high level of thermal energy. We need to ask if this is something which could be transferred from a prior universe, i.e. could there be a pop up nucleation effect, I.e. emergent space time? Appendix $\mathrm{H}$ gives a way for this to occur. We will now examine a mechanism which would allow for this to happen. It involves transfer of energy from a prior to the present universe.

\section{Appendix H: The D'Albembertain Operation in an Equation of Motion for Emergent Scalar Fields}

We begin with the D'Albertain operator as part of an equation of motion for an emergent scalar field. We refer to the Penrose potential (with an initial assumption of Euclidian flat space for computational simplicity) to account for, in a high temperature regime an emergent non-zero value for the scalar field $\phi$ due to a zero effective mass, at high temperatures [64].

When the mass approaches far lower values, it, a non-zero scalar field re appears.

Leading to $\phi \underset{T \rightarrow 2.7 \text { Kelvin }}{\longrightarrow} \varepsilon^{+} \approx 0^{+}$as a vanishingly small contribution to cosmological evolution

Let us now begin to initiate how to model the Penrose quintessence scalar field evolution equation. To begin, look at the flat space version of the evolution equation

$$
\ddot{\phi}-\nabla^{2} \phi+\frac{\partial V}{\partial \phi}=0
$$

This is, in the Friedman-Walker metric using the following as a potential system to work with, namely:

$$
\begin{aligned}
V(\phi) & \sim-\left[\frac{1}{2} \cdot\left(M(T)+\frac{\mathfrak{R}}{6}\right) \phi^{2}+\frac{\tilde{a}}{4} \phi^{4}\right] \\
& \equiv-\left[\frac{1}{2} \cdot\left(M(T)+\frac{\kappa}{6 a^{2}(t)}\right) \phi^{2}+\frac{\tilde{a}}{4} \phi^{4}\right]
\end{aligned}
$$

This is pre supposing $\kappa \equiv \pm 1,0$, that one is picking a curvature signature which is compatible with an open universe.

That means $\kappa=-1,0$ as possibilities. So we will look at the $\kappa=-1,0$ values. We begin with.

$$
\begin{aligned}
& \ddot{\phi}-\nabla^{2} \phi+\frac{\partial V}{\partial \phi}=0 \\
& \Rightarrow \phi^{2}=\frac{1}{\tilde{a}} \cdot\left\{c_{1}^{2}-\left[\alpha^{2}+\frac{\kappa}{6 a^{2}(t)}+M(T)\right]\right\} \\
& \Leftrightarrow \phi \equiv \mathrm{e}^{-\alpha \cdot r} \exp \left(c_{1} t\right)
\end{aligned}
$$


We find the following as far as basic phenomenology, namely

$$
\begin{aligned}
& \phi^{2}=\frac{1}{\tilde{a}} \cdot\left\{c_{1}^{2}-\left[\alpha^{2}+\frac{\kappa}{6 a^{2}(t)}+\left(M(T) \approx \varepsilon^{+}\right)\right]\right\} \\
& \underset{M(T \sim \text { high }) \rightarrow 0}{\longrightarrow} \phi^{2} \neq 0 \\
& \phi^{2}=\frac{1}{\tilde{a}} \cdot\left\{c_{1}^{2}-\left[\alpha^{2}+\frac{\kappa}{6 a^{2}(t)}+\left(M(T) \neq \varepsilon^{+}\right)\right]\right\} \\
& \underset{M(T \sim \text { Low }) \neq 0}{\longrightarrow} \phi^{2} \approx 0
\end{aligned}
$$

The difference is due to the behavior of $M(T)$. We use $M(T) \sim$ axion mass $m_{a}(T)$ in asymptotic limits with

$$
m_{a}(T) \cong 0.1 \cdot m_{a}(T=0) \cdot\left(\Lambda_{Q C D} / T\right)^{3.7}
$$

\section{Appendix I: Essential Gravitational Astronomy Considerations}

The experimental gravity considerations are covered in [65] [66] [67], and [68], and the idea should be especially to in our work to examine if [65] and [67] in terms of gravity are adhered to. As these are LIGO projects, we should be looking to see if what we are doing contravenes or backs the post Newtonian approximations of physics, so brought up.

Reference [67] is a must to review. In it, Corda reviews GR tests and our document must not contravene these basics. Can we obtain through our representation of gravitons, confirmation, or refutation of if the data sets are in adherence, or partially refute General Relativity. As far as [68], in terms of quantum cosmology, it is another similar parallel development to the ideas raised here. I urge readers to investigate it.

Finally, in lieu of [68] the author urges readers to look at the following quote from [69] page, 6 of that document ([69]).

Quote

In essence, for making a consistent cosmology, our results argue in favor of a string theory style embedding of the start of inflation and what we have argued so far is indicating how typical four-dimensional cosmologies have serious mathematical measure theoretic problems. This quantum measure theoretic problem are unphysical especially in light of the Stoica findings.

End of quote

This is a fairly consistent edorsement to the idea of what was presented for our model, starting with $H=0$ (no initial expansion) to the jump toward massive expansion, We urge the readers to review it, as well as to review what Corda brought up in [67].

As to [69] as it is, is a summary of what the author views as to what would be foundational investigation of gravity, and to see if it can be made in adherence to GR. That plus Appendix A of [70]. 